\title{
A Preocupação com a Sustentabilidade na Ótica de Alunos, Docentes e Funcionários de uma Universidade Brasileira
}

\author{
The Concern with Sustainability from the View of Students, Teachers and Employees of \\ a Brazilian University
}

\section{La Preocupación por la Sostenibilidad desde la Visión de Estudiantes, Profesores y Funcionarios de una Universidad Brasileña}

Thiago Antônio Beuron ${ }^{1}$ tbeuron@gmail.com http://lattes.cnpq.br/3128023764035312 https://orcid.org/0000-0002-7254-9145

Gustavo da Rosa Borges ${ }^{1}$ gustavodarosaborges@gmail.com http://lattes.cnpq.br/5162584966597575 https://orcid.org/0000-0002-9869-376X

Sávio Costa Borges ${ }^{1}$ saviocb@outlook.com http://lattes.cnpq.br/3455466905724491 https://orcid.org/0000-0002-3789-332X

Carine Hernandes Castilho ${ }^{3}$ carinehcastilho@hotmail.com http://lattes.cnpq.br/2863086442009851 https://orcid.org/0000-0001-6677-3880

Valeria Garlet ${ }^{1}$ valeriagarlet@yahoo.com.br http://lattes.cnpq.br/6442130419963857 https://orcid.org/0000-0001-9606-6001

Lucia Rejane da Rosa Gama Madruga² lucia.rejane@hotmail.com http://lattes.cnpq.br/6101538332418762 https://orcid.org/0000-0001-8571-8203

Universidade Federal do Pampa, UNIPAMPA, Brasil ${ }^{1}$ Universidade Federal de Santa Maria, UFSM, Brasil ${ }^{2}$ Centro Universitário da Região da Campanha, URCAMP, Brasil ${ }^{3}$

Recebido em: 21/07/2020 / Revisão: 01/08/2020 / Aprovado em: 25/09/2020

Editores responsáveis: Prof. Dr. Antônio Giovanni Figliuolo Uchôa e Prof. Dr. Jonas Fernando Petry

Processo de Avaliação: Double Blind Review DOI: https://10.47357/ufambr.v2i3.7913 
A Preocupação com a Sustentabilidade na Ótica de Alunos, Docentes e Funcionários de uma Universidade Brasileira

\title{
Resumo
}

Este estudo tem o intuito verificar se existe diferença de preocupação ambiental de acordo com o perfil de pessoas que frequentam o ambiente acadêmico. Para isso, foi mensurada a preocupação ambiental de alunos, docentes e funcionários de uma universidade no Brasil. Realizou-se uma survey com a participação de 1.070 indivíduos e percebeu-se que em geral, alunos, docentes e funcionários se preocupam com a sustentabilidade. Sobre as diferenças perceptivas, descobriu-se por meio de Teste-T e Anova que discentes apresentaram menor nível de preocupação em relação a docentes e funcionários. As mulheres apresentaram maior preocupação ambiental, e indivíduos ainda sem formação superior possuem menor nível preocupação ambiental. No que se refere à idade, o estudo mostrou com base na Regressão Linear que houve baixa influência na preocupação ambiental, ou seja, não é possível afirmar que a preocupação com a sustentabilidade aumente com o passar do tempo. Nota-se que a sustentabilidade ainda tem muito a ser discutida em todos os âmbitos, mas principalmente no ambiente acadêmico, lugar da construção do conhecimento e da difusão de atitudes e ações sustentáveis.

Palavras-chave: Conscientização Ambiental. Sustentabilidade. Universidades.

\section{The Concern with Sustainability from the View of Students, Teachers and Employees of a Brazilian University}

\begin{abstract}
This study item or intuitive to verify if there is a difference of environmental concern according to the profile of people who frequent or academic environment. For isso, it was measured to the environmental concern of students, teachers and officials of a university in Brazil. A survey was carried out with the participation of 1,070 individuals and perceived that in general, students, teachers and officials are concerned with sustainability. Regarding the perceptual differences, it was discovered through Teste-T and Anova that students had a lower level of concern in relation to teachers and civil servants. As women have a higher environmental concern, and individuals have a higher level of education and have a lower level of environmental concern. Not that it refers to the idea, or I study based on the Linear Regression that it has a low influence on environmental concern, or that it is not possible to affirm that concern for sustainability increases as time passes. Note-I know that sustainability is still very much to be discussed in all areas, but mainly not academic environment, place of construction of knowledge and diffusion of sustainable attitudes and actions.
\end{abstract}

Keywords: Environmental Awareness. Sustainability. University.

\section{La Preocupación por la Sostenibilidad desde la Visión de Estudiantes, Profesores y Funcionarios de una Universidad Brasileña}

\section{Resumen}


A Preocupação com a Sustentabilidade na Ótica de Alunos, Docentes e Funcionários de uma Universidade Brasileira

Este estudio tiene como objetivo verificar si existe diferencia en la preocupación ambiental según el perfil de las personas que asisten al ámbito académico. Para ello, se midió la preocupación ambiental de estudiantes, profesores y empleados de una universidad en Brasil. Se realizó una encuesta con la participación de 1.070 personas y se notó que, en general, los estudiantes, docentes y empleados están preocupados por la sostenibilidad. En cuanto a las diferencias perceptivas, a través de Test-T y Anova se descubrió que los estudiantes mostraban un menor nivel de preocupación en relación a los profesores y empleados. Como las mujeres mostraron una mayor preocupación por el medio ambiente, y las personas sin educación superior tienen un menor nivel de preocupación por el medio ambiente. En cuanto a la edad, el estudio mostró con base en Regresión Lineal que hubo poca influencia en la preocupación ambiental, es decir, no es posible afirmar que la preocupación por la sostenibilidad aumente con el tiempo. Cabe señalar que la sostenibilidad aún tiene mucho que discutir en todos los ámbitos, pero principalmente en el ámbito académico, el lugar para la construcción del conocimiento y la difusión de actitudes y acciones sostenibles.

Palabras Clave: Conciencia Ambiental. Sustentabilidad. Universidades 
A Preocupação com a Sustentabilidade na Ótica de Alunos, Docentes e Funcionários de uma Universidade Brasileira

\section{INTRODUÇÃO}

Nos primórdios do século XXI, a problemática da sustentabilidade global tem sido difundida e reconhecida pelas lideranças mundiais e se tornado um tópico discutido por jornalistas, cientistas, professores, estudantes e demais integrantes da sociedade nas mais diversas partes do mundo (Adams, 2006). De modo geral, a sustentabilidade é uma temática relevante, sendo representada por meio de um equilíbrio entre os fatores sociais, ambientais e econômicos (Oliveira Filho, 2004; Sauvé, 2005; Finkbeiner et al., 2010; Pappas, 2012; Carroll \& Buchholtz, 2014).

A importância da discussão sobre sustentabilidade foi acentuada a partir do momento em que o homem percebeu que, apesar dos avanços tecnológicos, seria incapaz de satisfazer a demanda exponencial por produtos, em especial os dependentes de recursos naturais, os quais estão presentes em quantidade finita (Aguiar \& Caleman, 2013).

O aumento da população humana, juntamente com o avanço tecnológico, está ocasionando uma série de explorações aos recursos naturais e grandes impactos à sociedade em geral (Felix \& Dos Santos, 2013). Frente a esta indesejada situação, desde o início da década de 80, vem se discutindo como conciliar a exploração econômica com a conservação do meio ambiente (Adams, 2006).

Kuhlman e Farrington (2010) comentam que a sustentabilidade deve considerar o equilíbrio entre as exigências de uma mordomia de um lado e, do outro lado, o desejo por uma vida melhor. Nesta mesma linha, Pappas (2012) destaca que o ser humano deve dar mais atenção às necessidades coletivas que as individuais. Estes pensamentos fazem parte do chamado consumo sustentável (Wang et al., 2014).

Em relação ao comportamento, Wang et al. (2014) acreditam que o comportamento visando um consumo sustentável somente é possível se houver conhecimento e uma percepção sobre o valor ambiental. Uma das formas de provocar este maior conhecimento é por meio da educação formal, contando com a participação de professores, escolas e universidades (Sauvé, 2005; Gadotti, 2008; Pappas, 2012; Zsóka et al., 2013).

Para Sauvé (2005), os profissionais que atuam na área de educação ambiental vêm tomando consciência da importância e riqueza do projeto educativo que ajudaram a construir ao longo dos últimos trinta anos. Gadotti (2008) acredita na reforma educativa como processo para uma vida mais saudável e sustentável que poderá trazer o bem-estar a todos.

Mediante a importância que a educação possui para incentivar atos sustentáveis, alguns trabalhos buscam analisar se, de fato, alunos universitários vêm tendo uma preocupação ambiental. Em um dos poucos trabalhos encontrados, destaca-se o de Zsóka et al. (2013). Neste estudo, os autores constataram que alunos universitários possuem uma maior preocupação ambiental em comparação a alunos de ensino médio, sugerindo assim, que o ambiente universitário pode influenciar na personalidade. Para Howard e Sheth (1969), a personalidade, a classe social e o ambiente influenciam na predisposição e comportamento das pessoas. 
A Preocupação com a Sustentabilidade na Ótica de Alunos, Docentes e Funcionários de uma Universidade Brasileira

Além do perfil, o ambiente também influencia o modo de pensar e agir. Quandt (1956) descreveu que pessoas pensam e agem sob influências do ambiente. A partir disso, é provável que pessoas que frequentam um determinado ambiente tendem a pensar diferente das que frequentam outro ambiente. Em relação a esta configuração teórica, este estudo analisa se as pessoas que ocupam diferentes papeis em um mesmo ambiente (universitário) possuem distintas preocupações ambientais. Castilho, Nascimento e Quesada (2019) destacam que docentes devem impulsionar um papel ativo sustentável dentro das universidades, mas admitem que isto pode depender do nível de ensino de cada um. Neste caso, observa-se que diferentes perfis podem influenciar percepção da sustentabilidade, podendo fazer parte de cada agente da universidade: docentes, funcionários e alunos.

De forma geral, considera-se que alunos e demais pessoas que trabalham em universidades possuem diferentes perfis, sendo que a análise da relação entre estes diferentes perfis com a preocupação ambiental é um assunto pouco explorado pela literatura. Por outro lado, poucos trabalhos buscaram fazer esta verificação, se de fato, docentes, técncos e alunos possuem diferentes percepções sobre a preocupação ambiental. Buscando contribuir para esta lacuna, o presente artigo tem foco norteador a seguinte questão de pesquisa: existe diferença na preocupação com a sustentabilidade de acordo com o perfil de pessoas que frequentam o ambiente acadêmico?

Com isso, o objetivo do presente estudo é verificar se existe diferença de preocupação ambiental de acordo com o perfil de pessoas que frequentam o ambiente acadêmico. Cabe ressaltar que Zsóka et al. (2013) realizaram sua pesquisa apenas com alunos. Neste trabalho, serão incluídos além de alunos universitários, docentes e funcionários que trabalham na universidade, buscando o conhecimento das características de toda a comunidade acadêmica.

As seguintes seções abordaram uma revisão da literatura sobre sustentabilidade e conscientização ambiental. Na sequência, será apresentado o método do estudo, os resultados da pesquisa, as considerações finais e as referências utilizadas.

\section{REFERENCIAL TEÓRICO}

Como destacado, o referencial teórico do presente estudo será subdividido em sustentabilidade e consciência ambiental.

\subsection{A Sustentabilidade}

A origem de sustentabilidade popularizou-se a partir de um objetivo que as políticas públicas deveriam alcançar, inspirado no Relatório de Bundtland em 1987 (Kuhlman \& Farrington, 2010). O conceito de sustentabilidade surgiu para conseguir agregar o crescimento e a industrialização preservando o equilíbrio ambiental. Com o tempo, esse conceito evoluiu, sendo referido no Relatório Brundtland como um desenvolvimento que satisfaça as necessidades do presente sem comprometer a capacidade das gerações futuras realizarem, também, as suas necessidades (Adams, 2006).

Gadotti (2008) acredita que sustentabilidade seja o sonho de bem viver, sendo equilíbrio e harmonia com o meio em que se vive. Corroborando Kuhlman e Farrington (2010), a 
A Preocupação com a Sustentabilidade na Ótica de Alunos, Docentes e Funcionários de uma Universidade Brasileira

sustentabilidade vem a ser um conjunto de recursos naturais, qualidade do meio ambiente e capital que será deixado de legado para as gerações vindouras.

Buscando uma melhor compreensão sobre o assunto, Rattner (1999) aborda que a sustentabilidade não deve ser vista apenas como um enfoque que aborde o meio ambiente natural. Seus cernes estão localizados em um relacionamento interno à sociedade, de natureza econômica e politicamente equilibrada e igualitária.

Mediante estas diferentes concepções, Moacir (2005) corrobora com o entendimento de que há divergências sobre os conceitos entre desenvolvimento sustentável e humano, ao qual, a ideologia aborda separadamente pontos sociais e ambientais. Para Diegues (1992), os princípios ecológicos como conservação da biodiversidade e recursos naturais são ferramentas importantes para a reprodução das sociedades sustentáveis.

Estas diferentes abordagens são elencadas por alguns outros autores, como Oliveira Filho (2004), Sauvé (2005), Finkbeiner et al. (2010), Pappas (2012), Carroll e Buchholtz (2014). Além dos fatores sociais e ambientais, os mesmos autores ilustram a existência de um terceiro fator: econômico.

Na concepção de Oliveira Filho (2004), há uma necessidade de existir harmonia entre os fatores ambientais, sociais e econômicos. Corroborando com Oliveira Filho (2004), Finkbeiner et al. (2010) destaca que o conceito de sustentabilidade não deve estar atrelado apenas ao contexto de preservação do meio ambiente, mas que está alicerçado em três dimensões: ambiente, economia e bem-estar social, em que a população deve encontrar o seu equilíbrio.

Sauvé (2005) acredita que o meio ambiente possui as mais diversas riquezas não se limitando apenas ao sentido ecológico, mas também possui importantes características no conceito de desenvolvimento pessoal e social. Já para Pappas (2012), em virtude do agravamento das condições ambientais, sociais e econômicas, há uma necessidade de resposta da comunidade acadêmica, ou seja, o ensino superior tem papel vital na tarefa de ajudar a humanidade ter harmonia, tanto na esfera social, econômica e ambiental. Sobre estes três fatores, Carroll e Buchholtz (2014) conceituam a tríplice esfera da sustentabilidade composta pelos vieses ambiental, econômico e social, nos quais a economia trata de riquezas materiais; o social engloba a qualidade de vida e a equidade entre pessoas, comunidades e nações; e o contexto ambiental se refere à preservação das condições naturais do meio em que estamos inseridos.

Constata-se que a sustentabilidade deve abordar temas alusivos aos aspectos sociais, econômicos e ambientais. Neste mesmo raciocínio, Oliveira Filho (2004) complementa destacando que as variáveis sociais, econômicas e ambientais direcionam para um desenvolvimento.

O desenvolvimento sustentável seria, assim, aquele que satisfaça as necessidades do presente, sem comprometer a capacidade das futuras gerações atenderem suas próprias necessidades. De modo geral, o desenvolvimento sustentável viria a ser o que abrangesse as satisfações das necessidades atuais sem prejudicar as gerações futuras no seu sustento (Almeida, 2002). Para Jacobi et al. (2003), o desenvolvimento sustentável pode ser compreendido por um processo 
A Preocupação com a Sustentabilidade na Ótica de Alunos, Docentes e Funcionários de uma Universidade Brasileira

em que os pontos mais acentuados estão interligados com recursos e sua exploração além do direcionamento do desenvolvimento tecnológico.

Embora o desenvolvimento, de forma geral, seja almejado, Gadotti (2005) destaca que uma organização para se tornar sustentável deve minimizar os impactos sobre os recursos naturais, respeitando os limites ambientais, reduzindo seu consumo e tornando-se mais eficaz. Assim sendo, no entendimento de sustentabilidade, o desenvolvimento sustentável seria aquele que satisfaz as necessidades humanas sem comprometer as futuras gerações, buscando convergir o progresso econômico com o respeito às ações sociais e ambientais.

Desta forma, é importante as empresas tomarem consciência da importância do conceito de sustentabilidade dentro das corporações, sensibilizando seus agentes a respeito da importância de reduzir os impactos socioambientais e da oportunidade de investir em alternativas para sua redução (De Felix \& Dos Santos, 2013). No ambiente corporativo, a meta é criar valor para acionistas no longo prazo, aproveitando-se de oportunidades e gestão de riscos que estão relacionados com a economia e o desenvolvimento social e ambiental (Carroll \& Buchholtz, 2014).

Pode-se considerar que sustentabilidade e sobrevivência estão vinculadas na sua essência, visto que quando ampliamos o significado da palavra sobrevivência podemos associar ao grande poder de transformação de elementos naturais acumulados pelo ser humano (Almeida, 2002).

De modo geral, o significado de sustentabilidade está em harmonia com o princípio de que o crescimento e suas possibilidades necessitam obedecer a limites, os quais devem respeitar à existência de outros agentes sociais, as práticas educativas incluindo o diálogo e políticas de desenvolvimento, as quais respeitam dimensões culturais, ecológicas e humanas. (Jacobi et al., 2003).

Entretanto, Martins (2005) entende que a sustentabilidade ambiental necessita de uma compreensão por parte da sociedade em geral, como fonte de alternativa para encontrar soluções que visem atender às necessidades sem agredir o meio em que vivemos, proporcionando uma qualidade de vida melhor e mais saudável. A natureza vem sofrendo vastas degradações pelas corporações as quais delimitam os recursos naturais para o desenvolvimento biológico da civilização, sobrevivência e conforto da sociedade em geral possibilitando o aumento de impactos sobre ela e reduzindo o equilíbrio ambiental (Guimarães, 2007).

Uma das formas de despertar um melhor equilíbrio ambiental é objetivar a redução do consumo. Acredita-se que o consumo sustentável tende a ser influenciado pelas culturas, como por exemplo, no Brasil, que segundo os autores a conscientização de um consumo ético origina-se mais do estado e politicas públicas do que da sociedade organizada (Ariztía, 2014).

O consumo e a produção insustentável são padrões que foram adotados pelo ser humano e que podem levar a desastres de proporções globais. Buscar alterações nesses padrões que levam a maximizar os impactos no meio ambiente é uma questão chave para cada país e população (Brizga et al., 2014). 
A Preocupação com a Sustentabilidade na Ótica de Alunos, Docentes e Funcionários de uma Universidade Brasileira

Para que a sustentabilidade de fato ocorra, é necessário que os agentes tenham uma visão global da busca por essa mudança de conceitos e ações por parte dos consumidores, empresas e governo (Aguiar \& Caleman, 2013). Portanto, estudar o comportamento de consumo tornase relevante para analisar a compreensão sobre os hábitos e intenções de consumo.

\subsection{Conscientização ambiental}

Embora recentemente a sustentabilidade seja compreendida como um princípio a ser buscado por todas as partes interessadas no contexto das organizações, ainda existe carência de metodologia para mensurar o desempenho da sustentabilidade, principalmente nas dimensões econômica e social (Finkbeiner et al., 2010).

Baumgarten (2002) acredita que o avanço dos comércios e dos sistemas produtivos seja para objetivar a satisfação dos produtores ou dos consumidores. Assim sendo, a natureza passa a ser vista como meio de exploração para a lucratividade e bem-estar da sociedade e não como uma fonte de recursos naturais para a qualidade de vida. Por outro lado, De Medeiros et al. (2014) destacam que preencher todas as expectativas do consumidor é uma condição relevante entre os fatores críticos de sucesso para a consolidação de um produto sustentável.

Nidumolu, Prahalad e Rangaswami (2009) pontuam que há resistência no tocante à sustentabilidade nas empresas, já que algumas delas acreditam que uma maior devoção aos aspectos sustentáveis pode ocasionar perda de competitividade, aumentando os custos e não trazendo benefícios financeiros imediatos.

Dessa forma, parece que o primeiro ponto para fazer com que pessoas entendam e reflitam sobre a sustentabilidade na sua vida, trabalho e sociedade seja a conscientização. Conscientizar é um pressuposto relevante para que ocorram princípios ecológicos dentro das organizações, nas quais é um tema cada vez mais discutido no ambiente corporativo de várias empresas globais, embora para alguns ainda seja visto como um simples fator de melhora no relacionamento em vez de uma forma que realmente transforme a dinâmica dos negócios (Adams, 2006).

Com intuito de alcançar a conscientização, Jacobi (2005) destaca a importância da colaboração dos educandos para a transformação da coletividade, visando um acordo para o bem comum, tendo em prática a busca por um ambiente mais equilibrado e sustentável. Gadotti (2008) diz que a preservação do meio ambiente depende de um conjunto de conscientização ecológica e educacional, ao qual entra a ecopedagogia como forma de aprendizagem e conscientização promovendo a educação e o bem estar entre todos.

Buscando analisar a conscientização e a contribuição da educação para que ocorra a sustentabilidade torna-se preponderante destacar que a educação ambiental exerce uma maior influência na mudança do comportamento das pessoas, inclusive em alunos universitários (Zsóka et al., 2013). Indo ao encontro desta evidência, Ontong e Grange (2018) realizaram um estudo com professores de escolas na África do Sul e constataram a importância do professor para despertar a consciência ambiental de alunos e da sociedade. 
A Preocupação com a Sustentabilidade na Ótica de Alunos, Docentes e Funcionários de uma Universidade Brasileira

Em relação à da educação superior, a primeira tarefa para Pappas (2012), deve ser educar os alunos na definição adequada de sustentabilidade e numa abordagem teórica que os permita pensar sobre os problemas da sustentabilidade. Em relação ao comportamento de alunos sobre a temática, Zsóka et al. (2013) constataram que alunos universitários possuem maiores interesses pela temática ambiental do que alunos de ensino médio. Os autores acreditam que isto pode ocorrer pelo fato de estudantes de ensino médio tendem a ser mais induzidos ao marketing, e os de ensino superior, tendem a ter hábitos de consumo mais conscientes.

Ainda sobre pensamentos diversos, Ingalhalikar et al. (2014) têm apontado diferenças de pensamento entre homens e mulheres. Por estes motivos apontados, que se espera aqui, se pessoas de diferentes perfis, mesmo em um mesmo ambiente, possuem preocupação com a sustentabilidade. $\mathrm{O}$ modo como ocorreu a pesquisa será destacado a seguir.

\section{METODOLOGIA}

O presente estudo caracteriza-se por ser descritivo e quantitativo, tendo como base uma universidade do Sul do país

A Universidade objeto desse estudo é uma das maiores instituições de ensino privadas do país. Seu campus principal é localizado em São Leopoldo, no Estado do Rio Grande do Sul. Possui outras unidades nas cidades de Porto Alegre, Caxias do Sul, Bento Gonçalves, Santa Maria, Rio Grande e Canoas. Possui ainda, sedes na cidade de Florianópolis, no Estado de Santa Catarina, e em Curitiba, no Paraná. O foco desse levantamento foi o campus principal, formado por uma área preservada de mais de 90 hectares, composta por arroios, lagos e áreas verdes interligadas. Em 2004, a instituição recebeu a certificação internacional ISO 14001, sendo a primeira universidade da América Latina a atestar o comprometimento com o controle dos impactos ambientais de suas atividades, produtos e serviços. Além disso, a universidade em questão possui papel de destaque quando abordado o cuidado e preservação da água e a Responsabilidade Social.

Ao todo, a universidade possui mais de 960 funcionários, 950 professores e 30 mil alunos. Neste levantamento, foram consideradas coletas de dados com alunos, docentes e funcionários, contando com a participação de 1.070 indivíduos. Foi utilizada amostragem não probabilística e buscou-se investigar o conjunto de características individuais que formam a organização utilizando um levantamento com corte transversal e análise quantitativa (Hair Jr. et al., 2005).

O período de coleta junto à comunidade acadêmica compreendeu os meses de setembro a novembro de 2015 , foi adotado o método de survey. Os questionários foram disponibilizados online (pela internet), criados utilizando a ferramenta formulários no Google Docs ${ }^{\circledR}$ e foi enviado o link via e-mail para os respondentes, conforme a orientação da administração da universidade. Foram convidados a participar do estudo todos os professores, funcionários e alunos do Campus principalda universidade.

A preocupação com a sustentabilidade foi medida por uma pergunta: "avalie o seu grau de preocupação com a sustentabilidade". Os respondentes assinalaram sua resposta por meio de uma escala Likert de 10 pontos, sendo a resposta 1 referente a"sem preocupação" e a resposta 10 " muito preocupado(a) ". Também foi analisado o perfil dos participantes do estudo por

UFAMBR, Manaus, v. 2, n. 3, art. 2, pp. 20-34, julho-dezembro, 2020 ttp://www.periodicos.ufam.edu.br/ufambr 
A Preocupação com a Sustentabilidade na Ótica de Alunos, Docentes e Funcionários de uma Universidade Brasileira

meio de seis questões, abordando o sexo, idade, estado civil, renda familiar, grau de instrução e atuação na universidade.

Para a apreciação dos dados, realizou-se análise de frequência e média. Além destas análises descritivas, utilizou-se Teste-T para analisar a diferença de médias de preocupação com a sustentabilidade entre os sexos, e Anova para analisar a diferença de média da preocupação com a sustentabilidade entre os diferentes estados civis, diferentes rendas familiares, diferentes grau de instrução e diferentes atuações na universidade. Por fim, realizou-se regressão linear simples para verificar se quanto maior a idade, maior seria o grau de preocupação com a sustentabilidade adotando-se os preceitos de Hair Jr. et al. (2009).

Os resultados da coleta dos dados e dos testes estatísticos implantados são demonstrados na seção que segue.

\section{APRESENTAÇÃO E DISCUSSÃO DOS RESULTADOS}

Neste capítulo serão apresentados e discutidos os resultados da pesquisa, inicialmente é apreciado o perfil dos participantes do estudo (Tabela 1).

Tabela 1: Perfil dos Participantes do Estudo

\begin{tabular}{|c|c|c|}
\hline Sexo & Frequência & Percentual \\
\hline Feminino & 641 & 59,9 \\
\hline Masculino & 429 & 40,1 \\
\hline Estado Civil & Frequência & Percentual \\
\hline Solteiro & 666 & 62,2 \\
\hline Casado & 347 & 32,4 \\
\hline Divorciado & 21 & 2,0 \\
\hline Outro & 36 & 3,4 \\
\hline Renda Familiar & Frequência & Percentual \\
\hline Classe E: até $\mathrm{R} \$ 1.085,00$ & 79 & 7,4 \\
\hline Classe D: de $\mathrm{R} \$ 1.085,00$ a $\mathrm{R} \$ 1.734,00$ & 166 & 15,5 \\
\hline Classe C: de $\mathrm{R} \$ 1.734$ a $\mathrm{R} \$ 7.475,00$ & 585 & 54,7 \\
\hline Classe B: de $\mathrm{R} \$ 7.475,00$ a $\mathrm{R} \$ 9.745,00$ & 87 & 8,1 \\
\hline Classe A: Acima de R $\$ 9.745,00$ & 153 & 14,3 \\
\hline Grau de instrução & Frequência & Percentual \\
\hline Superior Incompleto & 816 & 76,3 \\
\hline Superior Completo & 62 & 5,8 \\
\hline Pós-graduação & 192 & 17,9 \\
\hline Atuação & Frequência & Percentual \\
\hline Aluno & 846 & 79,1 \\
\hline Docente & 104 & 9,7 \\
\hline Técnico & 56 & 5,2 \\
\hline Aluno e técnico & 62 & 5,8 \\
\hline Técnico e docente & 1 & 0,1 \\
\hline Aluno e docente & 1 & 0,1 \\
\hline Média de idade & \multicolumn{2}{|c|}{28 anos } \\
\hline
\end{tabular}

Ao analisar a Tabela 1, verifica-se que a maioria dos participantes é do sexo feminino, solteiro(a), ocupante da classe $\mathrm{C}$ e aluno(a) que ainda não concluiu o ensino superior.Na sequência analisou-se a mensuração da preocupação com a sustentabilidade. 
A Preocupação com a Sustentabilidade na Ótica de Alunos, Docentes e Funcionários de uma Universidade Brasileira

Tabela 2: Preocupação com a Sustentabilidade

\begin{tabular}{c|c|c|c|c|c}
\hline Variável & Amostra & Mínimo & Máximo & Média & Desvio padrão \\
\hline Preocupação com a sustentabilidade & 1070 & 1,0 & 10,0 & $\mathbf{8 , 3 8 2}$ & 1,5877 \\
\hline \multicolumn{7}{c}{ Fonte: elaborado pelos autores }
\end{tabular}

Por meio da Tabela 2, pode-se observar que de modo geral, os participantes do estudo se preocupam com a sustentabilidade, já que a média geral foi de 8,382, em uma escala de 1 a 10. Este resultado apoia o entendimento de Pappas (2012) e Wang et al. (2014), os quais destacam que o ser humano deve dar mais atenção às necessidades coletivas que as individuais.

Embora a preocupação com a sustentabilidade, de modo geral, tenha ocorrido para os participantes do estudo, buscou-se identificar também se essa preocupação é mais ou menos intensa de acordo o perfil das pessoas. Para responder a esta indagação, realizaram-se testes estatísticos com intuito de comparar o perfil com o nível de preocupação ambiental. Os resultados destes testes são demonstrados na Tabela 3.

Tabela 3: Avaliação da Preocupação Ambiental de Acordo com o Perfil

\begin{tabular}{|c|c|c|c|c|}
\hline Sexo & Média & Desvio Padrão & Significância & Teste \\
\hline $\begin{array}{l}\text { Feminino } \\
\text { Masculino }\end{array}$ & $\begin{array}{l}8,50 \\
8,21\end{array}$ & $\begin{array}{l}1,54 \\
1,65\end{array}$ & $0,004 *$ & Teste-T \\
\hline Estado Civil & Média & Desvio Padrão & Significância & Teste \\
\hline $\begin{array}{l}\text { Solteiro } \\
\text { Casado } \\
\text { Divorciado } \\
\text { Outro } \\
\end{array}$ & $\begin{array}{l}8,35 \\
8,43 \\
8,06 \\
8,95 \\
\end{array}$ & $\begin{array}{l}1,58 \\
1,55 \\
2,12 \\
1,07 \\
\end{array}$ & 0,195 & Anova \\
\hline Renda Familiar & Média & Desvio Padrão & Significância & Teste \\
\hline $\begin{array}{l}\text { Classe E: até } \mathrm{R} \$ 1.085,00 \\
\text { Classe D: de } \mathrm{R} \$ 1.085,00 \text { a } \mathrm{R} \$ 1.734,00 \\
\text { Classe C: de } \mathrm{R} \$ 1.734 \text { a } \mathrm{R} \$ 7.475,00 \\
\text { Classe B: de } \mathrm{R} \$ 7.475,00 \text { a } \mathrm{R} \$ 9.745,00 \\
\text { Classe A: Acima de } \mathrm{R} \$ 9.745,00\end{array}$ & $\begin{array}{l}8,70 \\
8,47 \\
8,29 \\
8,28 \\
8,54 \\
\end{array}$ & $\begin{array}{l}1,43 \\
1,44 \\
1,67 \\
1,48 \\
1,59 \\
\end{array}$ & 0,111 & Anova \\
\hline Grau de instrução & Média & Desvio Padrão & Significância & Teste \\
\hline $\begin{array}{l}\text { Superior Incompleto } \\
\text { Superior Completo } \\
\text { Pós-graduação }\end{array}$ & $\begin{array}{l}8,31 \\
8,70 \\
8,60 \\
\end{array}$ & $\begin{array}{l}1,62 \\
1,42 \\
1,47 \\
\end{array}$ & $0,017 *$ & Anova \\
\hline Atuação** & Média & Desvio Padrão & Significância & Teste \\
\hline $\begin{array}{l}\text { Aluno } \\
\text { Docente } \\
\text { Funcionários }\end{array}$ & $\begin{array}{l}8,30 \\
8,75 \\
8,77 \\
\end{array}$ & $\begin{array}{l}1,64 \\
1,31 \\
1,29 \\
\end{array}$ & $0,004 *$ & Anova \\
\hline
\end{tabular}

* Significante ao nível de 0,05 .

** Não analisada as pessoas que acumulavam atuação por ser amostra pequena.

Fonte: elaborado pelos autores

A Tabela 3 demonstra a diferença de médias de acordo com o perfil da amostra, com exceção da idade, que será analisada posteriormente. Das cinco comparações realizadas, constatou-se haver diferença estatística em três delas (Sig. <0,005).

Com isso, pode-se considerar que mulheres possuem uma maior preocupação ambiental. Este resultado pode estar amparado no estudo de Ingalhalikar et al. (2014), os quais descobriram que mulheres possuem maior cognição social que homens. 
A Preocupação com a Sustentabilidade na Ótica de Alunos, Docentes e Funcionários de uma Universidade Brasileira

Pessoas ainda sem formação superior possuem menor nível preocupação ambiental. Esta constatação confirma os achados de Zsóka et al. (2013) os quais constataram que alunos universitários possuem uma maior preocupação ambiental, em comparação a alunos de ensino médio.

Por outro lado, alunos possuem um nível de preocupação ambiental menor que docentes e funcionários. Isto pode ser explicado pelo fato de docentes terem uma maior formação que alunos, e funcionários, de modo geral, um maior convívio com a sustentabilidade, pelo fato de trabalharem em uma instituição de ensino que busca atuar de forma sustentável, destacando a importância da universidade para a formação de uma maior preocupação com a sustentabilidade.

A partir disso, os resultados também apoiam o entendimento de Gadotti (2008), o qual propõe que a preocupação com a sustentabilidade depende de um conjunto de conscientização ecológica e educacional, no qual entra a ecopedagogia como forma de aprendizagem e conscientização promovendo a educação e o bem-estar entre todos.

Além de Gadotti (2008), Sauvé (2005), Pappas (2012) e Zsóka et al. (2013) também enfatizam que uma das formas de provocar este maior conhecimento é por meio da educação formal, contando com a participação de professores, escolas e universidades.

Estes resultados remetem à ideia de que a formação e o ambiente influenciam na preocupação ambiental já que, de modo geral, docentes possuem um alto nível de escolaridade e os funcionários da instituição, participam de cursos e são incentivados a desenvolverem preocupação ambiental. Esta possibilidade de relação não é recente em termos de indicação teórica, já que Quandt (1956) já havia descrito que as preferências e escolhas são influenciadas pelo ambiente.

A última análise comparativa foi em relação à idade. Como a idade foi uma variável informada pelos participantes, verificou-se que ela é uma variável métrica. Para isso, utilizouse regressão, sendo a idade a variável independente e a preocupação ambiental a variável dependente (Tabela 4).

Tabela 4: Relação entre Idade e Preocupação com a Sustentabilidade

\begin{tabular}{|c|c|c|c|c|c|c|c|c|}
\hline & \multirow{2}{*}{ Modelo } & \multicolumn{2}{|c|}{ Coeficientes não padronizados } & \multirow{2}{*}{$\begin{array}{c}\begin{array}{c}\text { Coeficientes } \\
\text { padronizados }\end{array} \\
\text { Beta }\end{array}$} & \multirow{2}{*}{$\mathbf{t}$} & \multirow{2}{*}{ Sig. } & \multirow{2}{*}{ VIF } & \multirow[t]{2}{*}{$\begin{array}{l}\text { Durbin- } \\
\text { Watson }\end{array}$} \\
\hline & & $\mathbf{B}$ & Modelo padrão & & & & & \\
\hline \multirow{2}{*}{1} & (Constante) & 8,074 & 0,149 & - & 54,358 & 0,000 & \multirow{2}{*}{1,00} & \multirow{2}{*}{0,06} \\
\hline & Idade & 0,011 & 0,005 & 0,067 & 2,194 & $0,028^{*}$ & & \\
\hline
\end{tabular}

a. Variável dependente: Preocupação com a sustentabilidade

* Significante ao nível de 0,05 .

Fonte: elaborado pelos autores

A Tabela 4 demonstra o resultado do Teste de Regressão. A significância de 0,028 e o Beta(B) positivo confirmam que quanto maior a idade, maior a preocupação ambiental. Entretanto a idade influencia pouco na preocupação ambiental, visto que o $\mathrm{R}$ foi de 0,067 , uma relação muito baixa na interpretação de Bisquerra, Sarriera e Martínez (2004). 
Um valor de R baixo apresentando relação é inusitado. Entretanto, analisou-se um baixo valor de VIF, o que, de acordo com Hair Jr. et al (2009), não apresentaria um problema de multicolinearidade. Por outro lado, o Durbin-Watson apresentou um resultado muito baixo $(0,06)$, o que, de acordo com Griffiths et al. (1993), refere-se a uma correlação espúria, o que prejudica a confiabilidade do resultado da regressão. Assim sendo, seguindo as indicações de Griffiths et al. (1993), não se pode afirmar uma relação da variável independente sobre a dependente. Com isso, entende-se que não exista a relação entre idade e preocupação ambiental, até mesmo, porque o $\mathrm{R}$ foi praticamente nulo. Portanto, não se pode afirmar que com o passar dos anos, as pessoas se preocupem mais com a sustentabilidade.

\section{CONSIDERAÇÕES FINAIS}

O objetivo do presente estudo foi verificar se existe diferença de preocupação ambiental de acordo com o perfil de pessoas que frequentam o ambiente acadêmico. Constatou-se que mulheres, pessoas com estudo mais elevado, de maior idade, funcionários e docentes possuem maior preocupação ambiental.

O fato de docentes e funcionários terem uma maior preocupação ambiental pode ser explicado por dois motivos: maior nível de escolaridade (Zsóka et al., 2013) ou ambiente de trabalho (Quandt, 1956; Carroll \& Buchholtz, 2014). Nesta mesma linha, observou-se que níveis de escolaridade mais elevado contribui para que haja maior percepção ambiental, sugerindo que universidades possuem de fato, um importante papel para despertar uma maior consciência ambiental (Para Felix, 2007; Ontong e Grange, 2018).

No que se refere ao sexo, não se observou outro trabalho similar que apontasse os resultados aqui encontrados, de que, mulheres possuem maior consciência ambiental. A explicação pode partir da sugestão de Howard e Sheth (1969), ao esclarecerem que a parecepção com a sustentabilidade depende da personalidade, da classe social e do ambiente; neste caso, as diferentes personalidades de sexo podem influenciar.

Em relação à idade, pessoas com maior idade possuem maior consciência ambiental e isto pode ser explicado pela maturidade, possivelmente, despertada pelo ambiente social (Quandt, 1956). Neste caso, reforça-se o papel da universidade; já que, as universidades promovendo maior consciência ambiental, acarretam em uma maior consciência social, que, tende a influenciar a comunidade em geral, mesmo as que não passaram pelas universidades.

Como contribuição científica, reforça-se a crença de que, pessoas com mais escolaridade e idade possuem maior consciência ambiental; e que, a percepção ambiental depende do perfil das pessoas. Um exemplo é o fato de mulheres terem maior percepção ambiental. Como contribuição prática, reforça-se a necessidade das universidades terem um papel ativo para despertar uma maior consciência ambiental na sociedade.

Em termos de limitação, o trabalho foi realizado por meio de amostra não probabilística e os resultados entrados limitam à universidade pesquisada, não sendo possíveis generalizações. Sugerem-se estudos em outros ambientes de ensino no sentido de verificar se de fato, alunos possuem menores níveis de consciência ambiental. Também, sugerem-se estudos para verificar se de fato, e por que, mulheres possuem maior consciência ambiental. 
A Preocupação com a Sustentabilidade na Ótica de Alunos, Docentes e Funcionários de uma Universidade Brasileira

\section{REFERÊNCIAS}

Adams, W. M. (2006, January). The future of sustainability: Re-thinking environment and development in the twenty-first century. In Report of the IUCN renowned thinkers meeting (Vol. 29, p. 31).

Aguiar, L. K., \& de Queiroz Caleman, S. M. (2013). Sustainability: Who is Driving it?. Desafio Online, 1(2), 81-104.

Almeida, F. (2009). O bom negócio da sustentabilidade. Rio de Janeiro: Ed. Nova fronteira.

Ariztia, T., Kleine, D., Maria das Graças, S. L., Agloni, N., Afonso, R., \& Bartholo, R. (2014). Ethical consumption in Brazil and Chile: Institutional contexts and development trajectories. Journal of Cleaner Production, 63, 84-92.

Baumgarten, M. (2002). Conhecimento, planificação e sustentabilidade. São Paulo em Perspectiva, 16(3), 31-41.

Bisquerra, R., Sarriera, J. C., \& Matínez, F. (2009). Introdução à estatística: enfoque informático com o pacote estatístico SPSS. Bookman Editora.

Brizga, J., Mishchuk, Z., \& Golubovska-Onisimova, A. (2014). Sustainable consumption and production governance in countries in transition. Journal of cleaner production, 63, 4553.

Carroll, A. B., \& Buchholtz, A. K. (2014). Business and society: Ethics, sustainability, and stakeholder management. Nelson Education.

Castilho, J. C. P., de Nascimento, R. M., \& Quesada, P. M. T. (2019). Projecto "com todos e para o bem de todos". Uma manifestação de extensão universitária sustentável na Escola Superior Pedagógica do Bié. Revista Angolana de Extensão Universitária, 1(1), 36-45.

da Costa Lima, G. F. (1997). O debate da sustentabilidade na sociedade insustentável. Revista De Ciências Sociais-Política \& Trabalho, v. 13, 201-222.

Felix, V. D. S., \& Santos, J. D. (2013). Gestão ambiental e sustentabilidade: um estudo de casos múltiplos no setor hoteleiro de João Pessoa/PB. Revista Eletrônica em Gestão, Educação e Tecnologia Ambiental, 10(10), 2185-17.

De Medeiros, J. F., Ribeiro, J. L. D., \& Cortimiglia, M. N. (2014). Success factors for environmentally sustainable product innovation: a systematic literature review. Journal of Cleaner Production, 65, 76-86.

Diegues, A. C. S. (1992). Desenvolvimento sustentável ou sociedades sustentáveis: da crítica dos modelos aos novos paradigmas. São Paulo em perspectiva, 6(1-2), 22-29.

Felix, R. A. Z. (2007). Coleta seletiva em ambiente escolar. REMEA-Revista Eletrônica do Mestrado em Educacao Ambiental, 18. 56-71.

Finkbeiner, M., Schau, E. M., Lehmann, A., \& Traverso, M. (2010). Towards life cycle sustainability assessment. Sustainability, 2(10), 3309-3322.

Gadotti, M. (2008). Educar para a Sustentabilidade. São Paulo: Instituto Paulo Freire.

Gadotti, M. (2005). Pedagogia da terra e cultura de sustentabilidade. Revista Lusófona de Educação, (6), 15-29.

Griffiths, W. E., Hill, R. C., \& Judge, G. G. (1993). Learning and practicing econometrics. John Willey and Sons. Inc. New York, USA.

Guimarães, M. (2007). Educação ambiental: participação para além dos muros da escola, p. 85-94. In.: De Mello, S. S., \& Trajber, Rachel [Coordenadoras]. Vamos Cuidar do Brasil: Conceitos e práticas em educação ambiental na escola, Brasília: Ministério da Educação, Coordenação Geral de Educação Ambiental: Ministério do Meio Ambiente, Departamento de Educação Ambiental: UNESCO.

UFAMBR, Manaus, v. 2, n. 3, art. 2, pp. 20-34, julho-dezembro, 2020 ttp://www.periodicos.ufam.edu.br/ufambr 
A Preocupação com a Sustentabilidade na Ótica de Alunos, Docentes e Funcionários de uma Universidade Brasileira

Hair, J. F., Black, W. C., Babin, B. J., Anderson, R. E., \& Tatham, R. L. (2009). Análise multivariada de dados. Porto Alegre: Editora Bookman.

Hair, J., Babin, B., Money, A., \& Samouel, P. (2005). Fundamentos de métodos de pesquisa em administração. Bookman Companhia Ed.

Howard, J. A., \& Sheth, J. N. (2001). A Theory of Buyer Behavior. Marketing: Critical Perspectives on Business and Management, 3, 81.

Ingalhalikar, M., Smith, A., Parker, D., Satterthwaite, T. D., Elliott, M. A., Ruparel, K., \& Verma, R. (2014). Sex differences in the structural connectome of the human brain. Proceedings of the National Academy of Sciences, 111(2), 823-828.

Jacobi, P. (2003). Educação ambiental, cidadania e sustentabilidade. Cadernos de pesquisa, (118), 189-206.

Jacobi, P. R. (2005). Educação ambiental: o desafio da construção de um pensamento crítico, complexo e reflexivo. Educação e pesquisa, 31(2), 233-250.

Kuhlman, T., Farrington, J. (2010). What is sustainability? Sustainability, 2(11), 3436-3448.

Martins, C. H., \& Oliveira, N. (2005). Indicadores econômico-ambientais na perspectiva da sustentabilidade. Porto Alegre: FEE.

Nidumolu, R., Prahalad, C. K., \& Rangaswami, M. R. (2009). Why sustainability is now the key driver of innovation. Harvard business review, 87(9), 56-64.

Oliveira Filho, J. E. (2004). Gestão ambiental e sustentabilidade: um novo paradigma ecoeconômico para as organizações modernas. Domus online, l(1).

Ontong, K., \& Le Grange, L. (2018). Exploring sustainability as a frame of mind: A multiple case study. South African Journal of Education, 38(1).

Pappas, E. (2012). A new systems approach to sustainability: University responsibility for teaching sustainability in contexts. Journal of Sustainability Education, 3(1), 3-18.

Quandt, R. E. (1956). A probabilistic theory of consumer behavior. The Quarterly Journal of Economics, 70(4), 507-536.

Rattner, H. (1999). Sustentabilidade-uma visão humanista. Ambiente \& sociedade, (5), 233240.

Sauvé, L. Educação Ambiental: possibilidades e limitações. Educação e Pesquisa, 31(2): 317$322,2005$.

Sterling, S. (2001). Sustainable education: re-visioning learning and change. (Schumacher Briefings No. 6). Green Books, Foxhole, Dartington.

Wang, P., Liu, Q., \& Qi, Y. (2014). Factors influencing sustainable consumption behaviors: a survey of the rural residents in China. Journal of Cleaner Production, 63, 152-165.

Zsóka, Á., Szerényi, Z. M., Széchy, A., \& Kocsis, T. (2013). Greening due to environmental education? Environmental knowledge, attitudes, consumer behavior and everyday proenvironmental activities of Hungarian high school and university students. Journal of Cleaner Production, 48, 126-138. 